\title{
The new Athens center on data processing from the neutron monitor network in real time
}

\author{
H. Mavromichalaki ${ }^{1}$, G. Souvatzoglou ${ }^{1}$, C. Sarlanis ${ }^{1}$, G. Mariatos $^{1}$, M. Gerontidou ${ }^{1}$, A. Papaioannou ${ }^{1}$, C. Plainaki $^{1}$, \\ S. Tatsis ${ }^{1}$, A. Belov ${ }^{2}$, E. Eroshenko ${ }^{2}$, and V. Yanke ${ }^{2}$ \\ ${ }^{1}$ University of Athens, Physics Department, Section of Nuclear and Particle Physics, Zografos 15771 , Athens, Greece \\ ${ }^{2}$ Institute of Terrestrial Magnetism, Ionosphere and Radio Wave Propagation (IZMIRAN), 142190, Troitsk, Russia
}

Received: 22 February 2005 - Revised: 8 June 2005 - Accepted: 15 June 2005 - Published: 22 November 2005

Part of Special Issue "1st European Space Weather Week (ESWW)"

\begin{abstract}
The ground-based neutron monitors (NMs) record galactic and solar relativistic cosmic rays which can play a useful key role in space weather forecasting, as a result of their interaction with interplanetary disturbances. The Earth's-based neutron monitor network has been used in order to produce a real-time prediction of space weather phenomena. Therefore, the Athens Neutron Monitor Data Processing Center (ANMODAP) takes advantage of this unique multi-directional device to solve problems concerning the diagnosis and forecasting of space weather. At this moment there has been a multi-sided use of neutron monitors. On the one hand, a preliminary alert for ground level enhancements (GLEs) may be provided due to relativistic solar particles and can be registered around 20 to $30 \mathrm{~min}$ before the arrival of the main part of lower energy particles responsible for radiation hazard. To make a more reliable prognosis of these events, real time data from channels of lower energy particles and Xray intensity from the GOES satellite are involved in the analysis. The other possibility is to search in real time for predictors of geomagnetic storms when they occur simultaneously with Forbush effects, using hourly, on-line accessible neutron monitor data from the worldwide network and applying a special method of processing. This chance of prognosis is only being elaborated and considered here as one of the possible uses of the Neutron Monitor Network for forecasting the arrival of interplanetary disturbance to the Earth. The achievements, the processes and the future results, are discussed in this work.
\end{abstract}

Keywords. Interplanetary physics (Cosmic rays; Energetic particles; Instruments and techniques)

Correspondence to: H. Mavromichalaki

(emavromi@cc.uoa.gr)

\section{Introduction}

Fundamental research of the cosmic ray variations and their relation to different parameters of solar activity leads to a possibility of their application to a modern and essential problem: monitoring and prediction of space weather by cosmic rays (CR), using on-line data. There are two types of space phenomena for which monitoring and forecasting are very important: the big space radiation storms caused by the solar cosmic rays accelerated to energies up to $15-20 \mathrm{GeV}$ during the great solar flares (Tylka, 2001), and the great interplanetary disturbances which produce large geomagnetic storms at the Earth (Dorman et al., 2003; Villoresi et al., 1995). Various methods and approaches, with an attempt to make such a prognosis reliable, have been elaborated on and discussed in different papers (Belov et al., 1995, 2003; Dorman et al., 1993, 2003; Bieber and Evenson, 1995; Dorman, 2003; Kudela et al., 2000).

To employ the cosmic ray data for the space weather problem, three important tasks need to be solved with the existing neutron monitor network: 1) providing the good quality, real-time data at every station in the Internet; 2) providing reliable access to these data, with the possibility of collecting them at one point (Center) for the joint processing; 3 ) adjusting the programs calculating the key parameters of the space weather to the quasi-real-time operation. After the IZMIRAN neutron monitor started to present continuous data in the Internet in 1997, many new stations were involved in this process (Mavromichalaki et al., 2001; 2004), and at the present, about twenty-five NM stations provide their data in real or quasi-real time, in digital and/or graphical form. The location of these stations on Earth is illustrated in Fig. 1. Nowadays,a number of special programs, such as the Global Survey Method (GSM), the Ring Station Method (RSM), and others, allowing for the derivation of the cosmic ray density, anisotropy and pitch angle distribution from the NM network data, are elaborated and used in the fundamental research. 
Table 1. List of real-time neutron monitor stations connected to the Athens Center.

\begin{tabular}{llrrrrr}
\hline Stations & Abbrev & Lat $\left(^{\circ}\right)$ & Long $\left(^{\circ}\right)$ & Alt $(\mathrm{m})$ & $\mathrm{H}_{0}(\mathrm{mb})$ & $\mathrm{R}_{c}(\mathrm{GV})$ \\
\hline APATITY & APTY & 67.55 & 33.33 & 177 & 977.80 & 0.55 \\
ATHENS & ATHN & 37.97 & 23.72 & 260 & 974.70 & 8.53 \\
BAKSAN & BKSN & 43.28 & 42.96 & 0 & 818.50 & 6.91 \\
BARENTSBURG & BRBG & 78.12 & 14.42 & 0 & 964.70 & 0.20 \\
CAPESCHMIDT & CAPS & 68.92 & 180.53 & 0 & 1021.30 & 0.52 \\
EREVAN & ERVN & 40.17 & 44.25 & 2000 & 798.30 & 7.36 \\
EREVAN-3 & ERV3 & 40.17 & 44.25 & 3200 & 683.90 & 7.36 \\
ESOI & ESOI & 33.30 & 35.79 & 2025 & 800 & 10.41 \\
FORTSMITH & FSMT & 60.00 & -112.00 & 0 & 996.10 & 0.30 \\
INUVIK & INVK & 68.35 & -133.72 & 21 & 1019.10 & 0.14 \\
IRKUTSK & IRKT & 52.47 & 104.03 & 433 & 965 & 3.49 \\
KIEL & KIEL & 54.34 & 10.13 & 54 & 981.40 & 2.36 \\
LOMNICKY STIT & LMKS & 49.20 & 20.22 & 2634 & 733 & 3.88 \\
McMURDO & MCMD & -77.85 & 166.72 & 48 & 985.10 & 0.00 \\
MOSCOW & MOSC & 55.47 & 37.32 & 200 & 991.90 & 2.30 \\
NORILSK & NRLK & 69.26 & 88.05 & 0 & 1015.30 & 0.53 \\
NOVOSIBIRSK & NVBK & 54.80 & 83.00 & 163 & 999.20 & 2.69 \\
NEWARK & NWRK & 39.68 & -75.75 & 50 & 1008.60 & 2.21 \\
OULU & OULU & 65.05 & 25.47 & 0 & 990.00 & 0.77 \\
SOUTH POLE & SOPO & -88.00 & 210.00 & 2820 & 687.70 & 0.05 \\
THULE & THUL & 76.50 & -68.70 & 260 & 1011.50 & 0.00 \\
TIXIE BAY & TXBY & 71.6 & 128.90 & 15 & 1019.90 & 0.43 \\
YAKUTSK & YKTK & 62.02 & 129.73 & 105 & 10.20 .70 & 1.55 \\
\hline
\end{tabular}

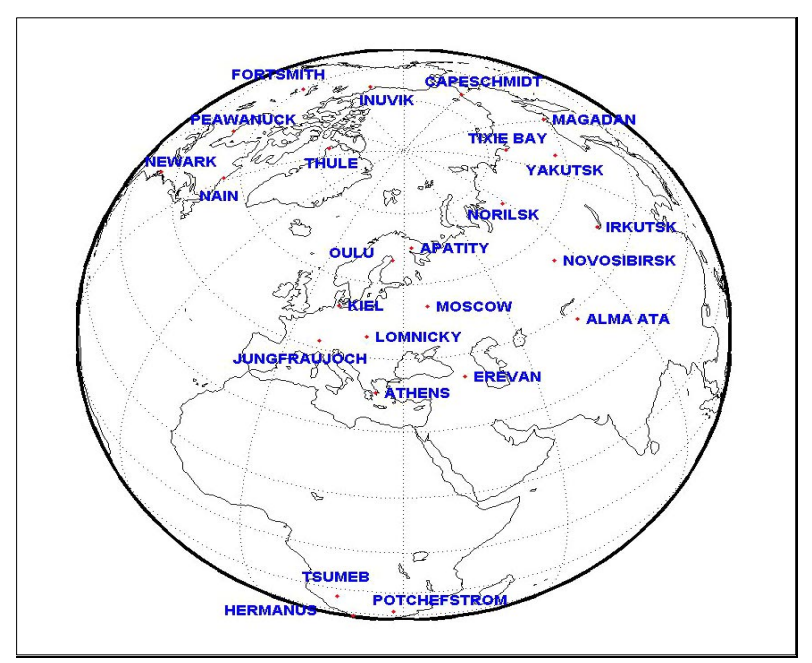

Fig. 1. A global distribution of all online Neutron Monitor stations.

The first steps in the process of collect data from a number of stations and analyzing them in real time have been made by the Bartol cosmic ray group (Bartol Research In- stitute - BRI) in the frame of the Space Ship Earth project (http://neutronm.bartol.udel.edu//). Then, a new real-time data collection system was developed by the IZMIRAN cosmic ray group, using the latest networking methods, in order to obtain data from the maximum possible number of stations, to provide sufficiently reliability of further analysis. The use of all stations as a unified, multi-directional detector makes the accuracy of the measurements substantially higher $(<0.1 \%$ for hourly data).

During the last two years the Athens Neutron Monitor Data Processing (ANMODAP) Center is being created at Athens University on the basis of the cosmic ray group activity.

\section{ANMODAP Center}

\subsection{Data collection system in Athens}

Accounting for the above-mentioned experience, in the Athens cosmic ray station a data collection system has been developed, with the capability of obtaining data from a large number of remote stations. The system is rather flexible and can be upgraded at any time. It is important to outline that the 


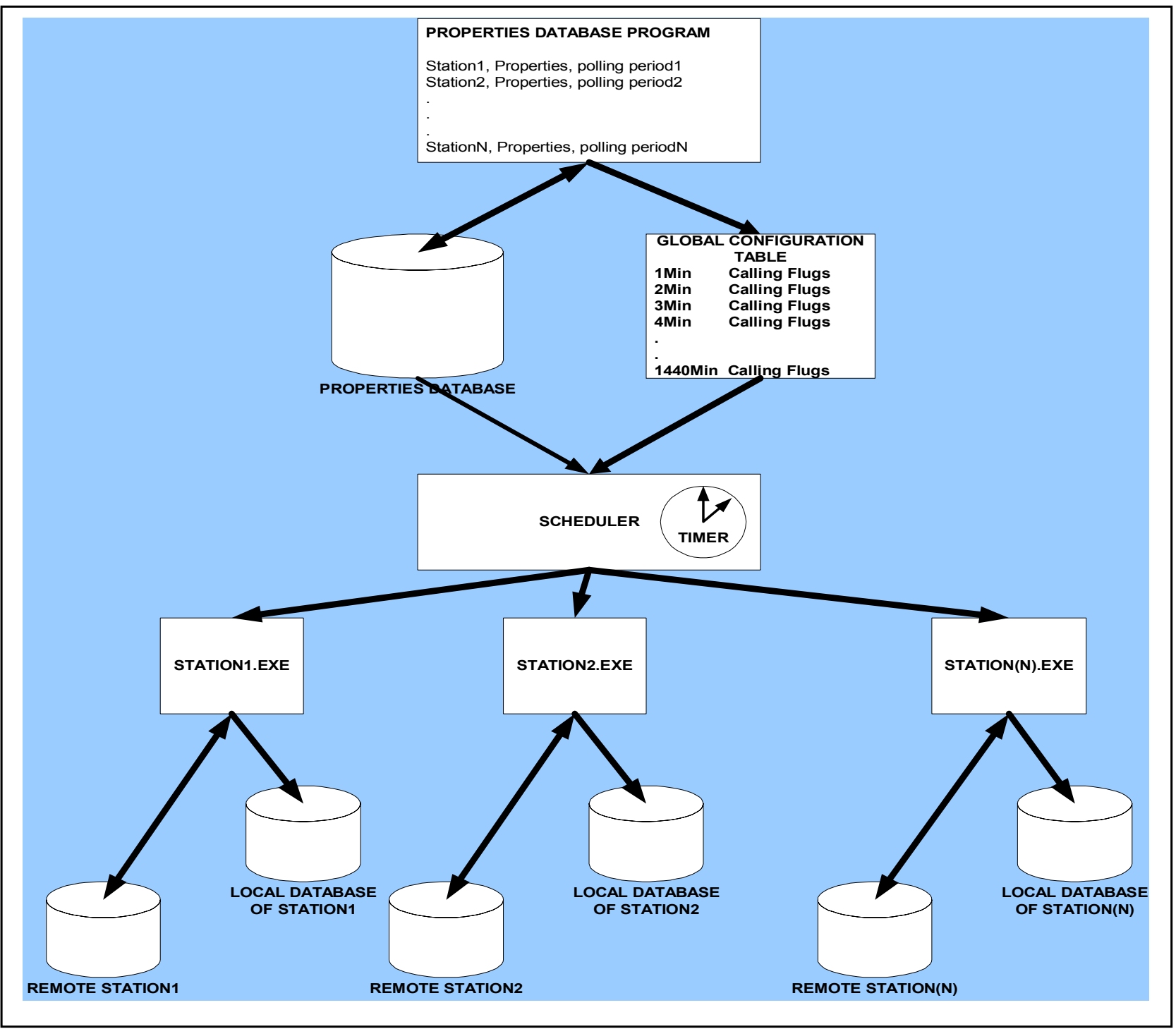

Fig. 2. A schematic diagram of the ANMODAP Center in real time.

system has the ability to provide reliable data, using independent programs of simultaneous data collection from different stations in a periodic scheme with a specific time period either automatically or even manually. Until today, there are 23 stations from which the described system could collect data. The characteristics of these NM stations (geographic coordinates, altitude, standard pressure, cut-off rigidity) are presented in Table 1 . However, up to now, only 19 of these stations are accessible online. In order to produce a continuous upgrade of the developed system, a number of algorithms and programs have been developed. The overall process of data can be seen in Fig. 2. The properties of every station from the network are input to one database program, the "Properties Database". Within the same database the initialization parameters of the data collection on poling are being deposed for every station. In particular, a "scheduler" algorithm has the ability to call various types of executable downloaders. A "Scheduler" program reads the properties of each station and makes a decision as to whether or not to make a data collection call to a station. For every different station there is a single data collection program which uploads data from a remote station to the local database of the network in Athens. For our study not only collecting data but also data presentation are very important. The neutron monitor recording system transfers one minute and hourly data to their server and refreshes their database every hour. A special program included in a 'scheduler' creates a graphical file once per hour which is displaced on the web page of the station presented at the server of the Athens Center. An advanced processing system of 1-, 5-, 15-, and 60-min data refreshes the database of every station, providing both graphical and digital form of presentation. Recently, an effort to combine the results of the NMN and those presented by other sources has been achieved, urging us into going forth 


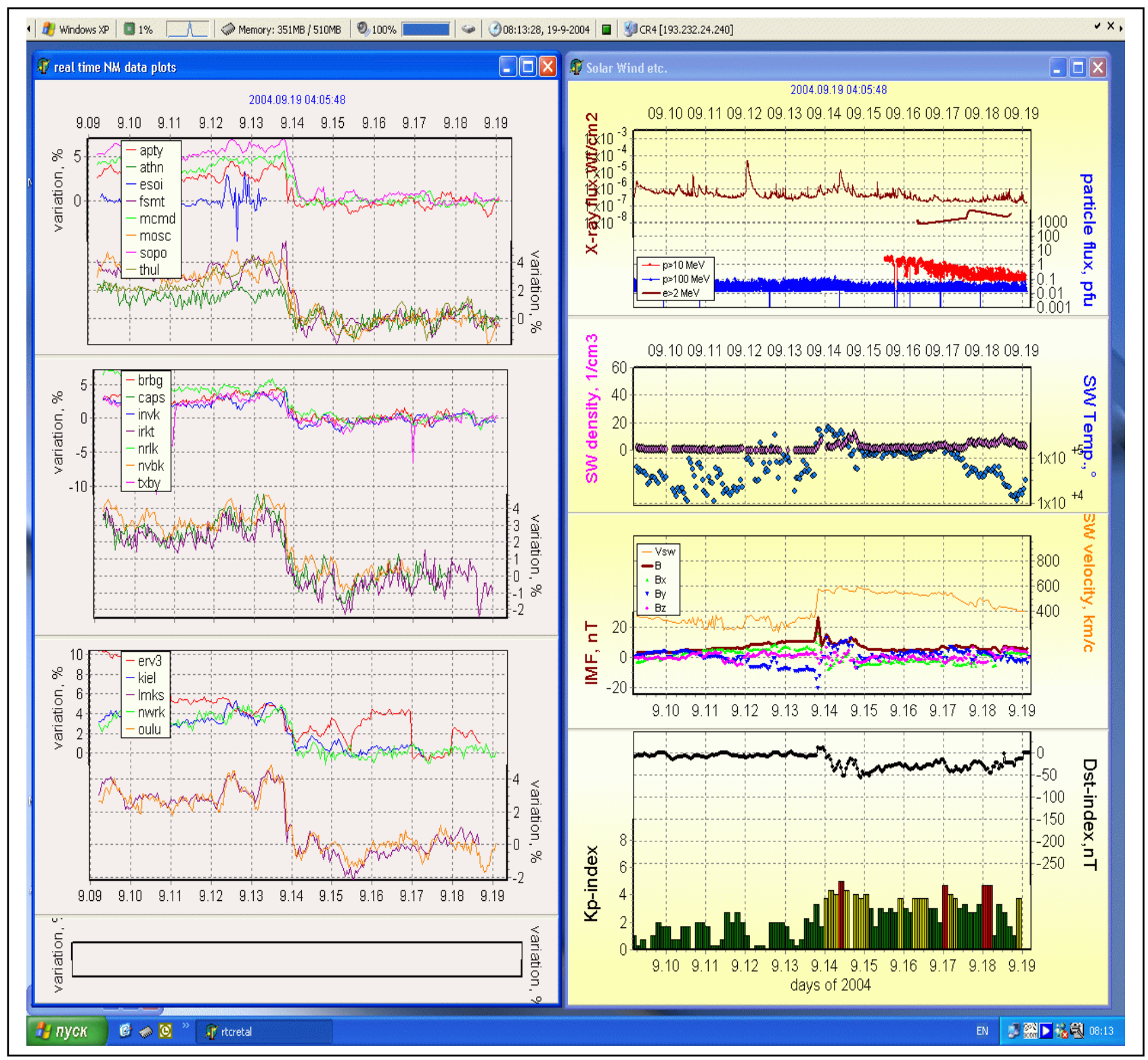

Fig. 3. Plots of real-time data from the NM Network (left panel) and from other sources (right panel).

with an even more detailed analysis. An example of the presentation of the collected data can be seen in Fig. 3, where, together with the data from different NMs, the various data from GOES and ACE satellites are presented online. An explanation of the relative parameters appearing on the graphs is given in Table 2.

\subsection{Applications of the Athens data processing center}

Collecting real-time data from the NM network can be used for diagnosing and forecasting aims, and are processed by the special programs on some key derived parameters. At this point the network data can be used in two directions: specifically first to a monitor and search for the Ground Level Enhancement (GLE) onset by the 1- and 5-min data and work- ing out the "Alert" signal in this connection, and second the $\mathrm{CR}$ variation monitoring by the hourly data and the searching for the predictors of the interplanetary shock arrival on the basis of CR density and anisotropy behavior.

\subsubsection{The GLE onset}

Some offers and possibilities for selecting the GLE onset are described in Dorman et al. (2004). A special program for searching the onset was elaborated by the Athens CR group. Data from at least three NM stations at Earth (two high latitudinal and one/two low latitudinal) and two independent satellite channels, for example, X-ray on GOES10 and GOES12, are processed every minute to search for the 
Table 2. Satellite data parameters being presented by the ANMODAP Center.

\begin{tabular}{ll}
\hline SATELLITE DATA & \\
Symbol & Explanation \\
\hline Solar wind temperature $(\mathrm{K})$ & ACE interplanetary ion temperature $(\mathrm{K})$ \\
Solar wind density $\left(\mathrm{cm}^{-1}\right)$ & ACE interplanetary proton density \\
Solar wind velocity $(\mathrm{km} / \mathrm{s})$ & ACE interplanetary bulk speed $(\mathrm{km} / \mathrm{s})$ \\
Interplanetary Magnetic Field B (nT) & ACE interplanetary magnetic field B in \\
& GSM coordintaes \\
IMF Bx component $(\mathrm{nT})$ & ACE interplanetary magnetic field $(\mathrm{nT})$ \\
IMF By component $(\mathrm{nT})$ & ACE interplanetary magnetic field $(\mathrm{nT})$ \\
IMF Bz component $(\mathrm{nT})$ & ACE interplanetary magnetic field $(\mathrm{nT})$ \\
$D_{s t}(\mathrm{nT})$ & Preliminary $D_{s t}$ index in nT \\
Particle Flux $(\mathrm{pfu})$ & GOES-10 particles at $>2 \mathrm{MeV}$ \\
Particle Flux $(\mathrm{pfu})$ & GOES-10 particles at $>10 \mathrm{MeV}$ protons \\
Particle Flux $(\mathrm{pfu})$ & GOES-10 particles at $>100 \mathrm{MeV}$ protons \\
X-ray flux $\left(\mu \mathrm{W} / \mathrm{m}^{2}\right)$ & GOES $12 \mathrm{X}$-ray at wavelength \\
& $=0.1-0.8 \mathrm{~nm}\left(\mu \mathrm{W} / \mathrm{m}^{2}\right)$ \\
$K_{p}$ & Preliminary 3-h $K_{p}$ index \\
\hline
\end{tabular}

Table 3. Results of the ALERT program on the event of 20 January 2005.

\begin{tabular}{lllll}
\hline 20 January 2005 & & & & \\
NM stations & Moscow & Kiel & Oulu & Norilsk \\
\hline Event started at & $6: 51$ UT & $6: 51$ UT & $6: 52$ UT & $6: 31$ UT \\
Established alert signal & $6: 55$ UT & $6: 55$ UT & $6: 56$ UT & $6: 35$ UT \\
Maximum of the event & $7: 05$ UT & $7: 05$ UT & $7: 00$ UT & $6: 41$ UT \\
\hline
\end{tabular}

start of the ground level enhancement. If the enhancement is found at least on two monitors and in X-ray channels simultaneously, it means the start of GLE, and our computer sends a signal to collect data from all neutron monitors throughout the network. The obtained alert signal is sent out as a forecasting signal by e-mail, and is mainly to run a system of minute data collection from the whole NM network, to be used in further, more complicated processing. In this case data on the network station are very important to be updated not less than every $5 \mathrm{~min}$. The number of necessary stations should be about 10-20. It may turn out to be that the NM network will be the only source of information during the powerful proton event because of the spoiled connection with the satellite.

Regarding the real-time searching for GLE onset we can say that for the event registered on 20 January 2005 our system was able to give an alarm with a $95 \%$ accuracy at approximately 5 to $10 \mathrm{~min}$ before the maximum of the event at the high latitude stations. The results of analysis of this event, after having applied our ALERT program, using data from four neutron monitor stations, are presented in Table 3. It is clearly seen that the obtained onset time from the ALERT program onset time is very close to the value calculated with the 2.5 sigma criterion (Dorman et al., 2004; Mavromichalaki et al., 2004). It is evidence of a good agreement between the onset times for the Moscow and Kiel stations. The event was registered at the Oulu NM station one minute later, whereas the Norilsk station recorded an increase twenty minutes earlier than the other three stations. In such cases of significant differences between the output onset times, an extra check should be made. In order to define whether the earliest onset time is a real ALERT or not, the data of the $100 \mathrm{MeV}$ proton channel should be analyzed. Our code is also being developed in this direction.

During the last two years GLEs Alerts were also estimated using this program. Usually we don't get false alarms. Taking into consideration that in the analysis the data from high latitude stations are included and also the fact that our program uses 5-min separate and X-ray data from GOES for a comparison, the probability that the observed increase is not associated with GLE is very low. 


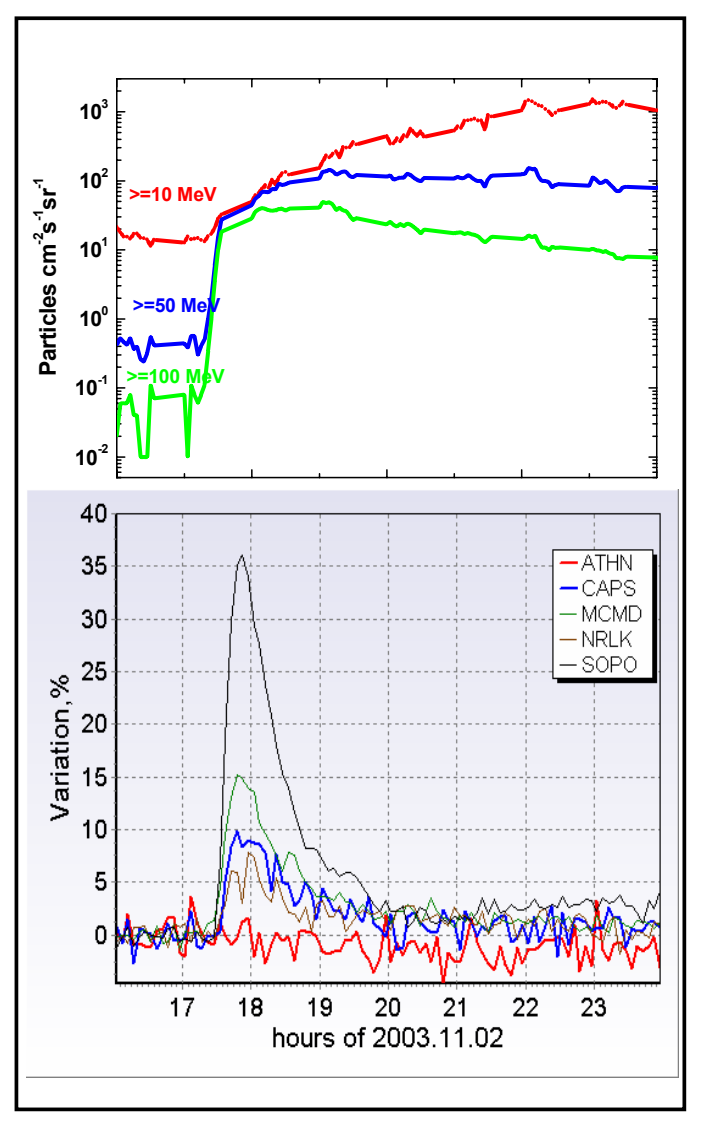

Fig. 4. The powerful SEP event on 2 November 2003.

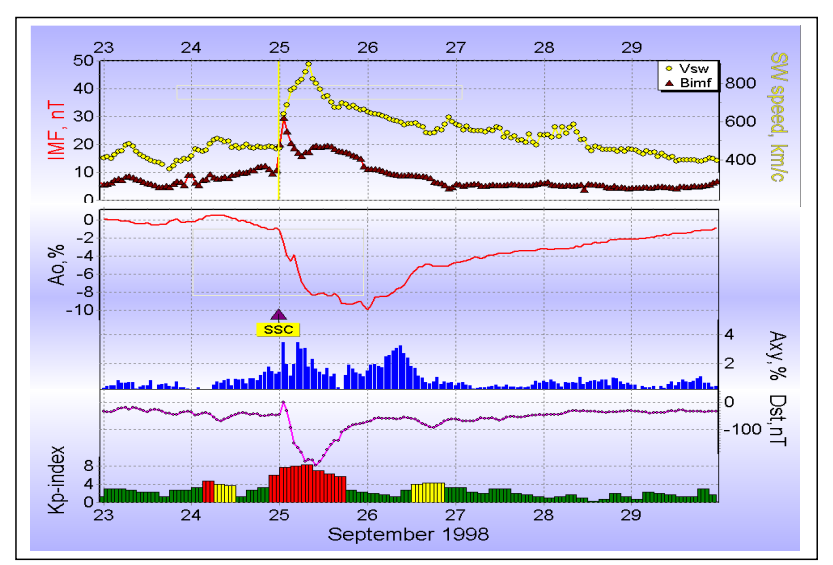

Fig. 5. A large heliospheric storm, as indicated by different space weather parameters.

In order to be accurate the real time algorithm takes different kinds of inputs from all the available sources. For big events the Alert stage is scheduled to be about $99 \%$ accurate. Our statistic is still poor because to the continuous upgrades of the input data source code in the program. It is possible that very soon we will present a full analysis of all the old available GLE data. It is well known that GLEs accompany only small fractions of the strong solar proton events and also that not all proton events result in ground enhancements, as well. However, a technique of calculating the relative flux increases using the data of high latitude stations eliminates the possibility of losing an event. An improved version of the ALERT program will be available soon via a link from the main page of the Athens Neutron Monitor station, visualizing all the forecasted events in real time.

\subsubsection{Prognosis of the low energy particle event evolving}

Solar relativistic particles registered at the Earth have an essential property to pro information on solar and interplanetary conditions much earlier than low- and mid-energy solar particles. Due to their big diffusion coefficient high-energy particles come from the Sun 8-20 min after the acceleration and escape into the solar wind, whereas the main part of lower energy particles, which cause dangerous situation for electronics and hazard radiation, usually come 30-60 min later. Proton events registered at the Earth (GLEs) have a complete profile well before the main development of the low-energy particle profiles. This is clearly seen in Fig. 4 with an example of the powerful SEP event on 2 November 2003. This fact can be used for the calculations of spectra and fluxes for lower energies at different levels 20-30 min after the onset by the ground level measurements (NMN), with an improvement of these results over the time. On the whole, it allows prognosis time behavior of non-relativistic solar protons up to 10-15 h (Dorman et al., 2004).

After the Alert is defined, our program sends a signal to collect data from all neutron monitors throughout the network. While the minute data are collected, the program of the proton enhancement analysis is running. It calculates spectra and other parameters for the estimation of the expected CR profiles for lower energies at different altitudes, several hours ahead. The proton spectrum will be derived with more and more accuracy along the accumulation of new and new data. This program is not realized yet, but it is under construction now.

\subsubsection{Geomagnetic storms}

A large heliospheric storm, indicated by different space weather parameters, is shown in Fig. 5, where significant variations in $\mathrm{CR}$ density and in the first harmonic of the CR anisotropy, derived from the NM network data, occurred simultaneously with dramatic changes in the interplanetary and geomagnetic parameters. Specific properties of the CR behaviour before the strong geomagnetic storms allow them to be used for the forecasting of the interplanetary disturbance arrival at the Earth (Belov et al., 1995; Ruffolo et al., 1999; Belov et al., 2001; Belov et al., 2003; Leerungnavarat et al., 2003 and references therein). So, another aspect of the real-time ground level monitoring of cosmic rays is to obtain galactic cosmic ray characteristics for diagnosing a situation in the heliosphere to predict some dangerous events in the Earth's vicinity. 
The proposed method on the prognosis of geomagnetic storms consists of the following steps:

- System of data collection in real time from a neutron monitor network has been created and supports an unlimited number of stations. In this listing high latitudinal stations, as well as mid- and low-latitudinal stations should be included.

- After collection data for a current hour from at least 15 stations (optimum 30) the program of analysis by the global survey method (GSM) (Belov et al., 2005) is started up. In result the CR density, the spectral parameters of density, and the three components of CR anisotropy vector are derived at this current hour.

- Amplitude of isotropic variation and characteristics of the CR anisotropy (first harmonic) are collected at the web site in real time and are the initial experimental data (so-called, key parameters) for analysis of the current heliospheric situation. To carry out more reliable analysis it would be desirable to use these results, together with relevant data on solar and solar wind measurements.

This program is now being revised by the collaboration of the IZMIRAN and ATHENS groups, through the current hourly data, coupling coefficients, and response functions, which were calculated earlier (Clem and Dorman, 2000; Villoresi et al., 2000) and tested. Of course, we use preliminary ("raw") data, and they are not always of good quality at the separate stations, as seen in Fig. 3. But the algorithm dismisses the data jumping if it exceeds three standard statistical errors at one or two stations.

\section{Conclusions}

The new Athens Neutron Monitor Data Processing Center based on the activity of the cosmic ray group at Athens University, provides real-time monitoring of cosmic ray variations. It was also created with the aim to make feasible the use of the neutron monitor network data in real time for the space weather tasks. The modern methods applied to these data give the possibility for forecasting the arrival at Earth of the powerful disturbances from the Sun. At present, twentyfive NM stations are operating continuously at different locations on the Earth and presenting their data in the Internet. The Athens Center, simultaneously with some other Centers (IZMIRAN, BARTOL University), gathers data to detect possible abrupt changes in the cosmic rays associated with the real solar wind and geomagnetic disturbances. This Center puts the basis of a world-wide data collection system in real time from the network and utilizes adjusted software capable of employing the real-time data for the space weather forecasting.

This Center offers a program with the possibility of successful prediction of the behavior of the low energy part of the SEP events near Earth by the ground-based cosmic ray measurements. The Center also accumulates experience and tested methods, and tries to elaborate on the more modern methods, in order to carry out a timely and feasible prognosis of the geomagnetic storms deriving precursors from the worldwide NM network.

By way of a conclusion we can say that the joint complex analysis of the relevant information from space-borne and ground-based detectors will minimize the number of false alarms and will maximize the reliability and the timely forecasting of the arrival of dangerous fluxes and disturbances from space.

Acknowledgements. Thanks are due to all NM groups for posting the data in the Internet and kindly provided these to the NM Network: Bartol Research Institute NSF grant ATM-0000315; IEP SAS in Kosice by VEGA grant 1147; Oulu; all Russian stations etc. This work is partly supported by PYTHAGOPAS II Project of the Greek Ministry of Education and by IZMIRAN RFFR grants 03-07-90389 and 04-02-16763. Thanks are also due to the anonymous referee for very useful remarks on this article. Many thanks to COST724 project supporting A. Papaioannou to present this work in the $1 \mathrm{st}$ European Space Weather week in Holland.

Topical Editor R. J. Forsyth thanks R. Pyle and another referee for their help in evaluating this paper.

\section{References}

Belov, A. V., Dorman, L., Eroshenko, E., Iucci, N., Villoresi, G., and Yanke, V.: Search for predictors of Forbush decreases, Proc. 24-th ICRC, 4, 888-891, 1995.

Belov, A. V., Bieber, J. W., Eroshenko, E. A., Evenson, P., Pyle, R., and Yanke, V. G.: Pitch-angle features in cosmic rays in advance of severe magnetic storms: Neutron monitor observations, Proc. 27 ICRC Hamburg 9, 3507-3510, 2001.

Belov, A. V., Bieber, J. W., Eroshenko, E. A., Evenson, P., Pyle, R., and Yanke, V. G.: Cosmic ray anisotropy before and during the passage of major solar wind disturbances, Adv. Space Res., 31, N4, 919-924, 2003.

Belov, A. V., Baisultanova, L. M., Eroshenko, E. A., Mavromichalaki, H., Yanke, V., Pchelkin, A., Mariatos, G., and Plainaki, C. Magnetospheric effects in cosmic rays during the unique magnetic storm in November 2003, J. Geophys. Res., in press, 2005.

Bieber, J. W. and Evenson, P.: Spaceship Earth - an optimized network of neutron monitors, Proc. 24th Intern. Cosmic Ray Conf., Rome, 4, 1316-1319, 1995.

Clem, J. and Dorman, L. I.: Neutron monitor response functions, Space Sci. Rev., 93, 1, 335-359, 2000.

Dorman, L. I., Iucci, N., and Villoresi, G.: The use of comic rays for continuous monitoring and prediction of some dangerous phenomena for the Earth's civilization, Astrophys. and Space Science, 208, 55-68, 1993.

Dorman, L. I.: Principles of cosmic ray using for space weather monitoring and forecasting, Proc. 28th Intern. Cosmic Ray Conf., Tsukuba, 7, 4269-4272, 2003.

Dorman, L. I., Belov, A. V., Eroshenko, E. A., Pustil'nik, A., Sternlieb, A., Yanke, V. G., and Zukerman, I.G.: Possible cosmic ray using for forecasting of major geomagnetic storms, accompanied by Forbush-effects, Proc. 28th Intern. Cosmic Ray Conf., Tsukuba, 6, 3553-3556, 2003. 
Dorman, L. I.: Cosmic Rays in the earth's atmosphere and underground, Kluwer Academic publishers, 2004.

Dorman, L. I., Pustilnik, L. A., Sternlieb, A., Zukerman, I. G., Belov, A. V., Eroshenko, E. A., Yanke, V. G., Mavromichalaki, H., Sarlanis, C., Souvatzoglou, G., Tatsis, S., Iucci, N., Villoresi, G., Fedorov, Yu., Shakhov, B. A., and Murat, M.: Monitoring and Forecasting of Great Solar Proton Events Using the Neutron Monitor Network in Real Time, IEEE for Plasma Science, 32, 1478-1488, 2004.

Kudela, K., Storini, M., Hoffer, M., and Belov, A.: Cosmic Rays in relation to space weather, Space Sci. Rev., 93, 153-174, 2000.

Leerungnavarat, K., Ruffolo, D., and Bieber, J. W.: Loss cone precursors to Forbush decreases and advance warning of space weather effects, The Astronomical J., 593, 587-596, 2003.

Mavromichalaki, H., Sarlanis, C., Souvatzoglou, G., Tatsis, S., Belov, A., Eroshenko, E., Yanke, V., and Pchelkin, A.: Athens Neutron Monitor and its aspects in the cosmic-ray variations studies, Proc. 27th ICRC Hamburg, 10, 4099-5004, 2001.
Mavromichalaki, H., Yanke, V., Dorman, L., Iucci, N., Chillingaryan, A., and Kryakunova, O.: Neutron Monitor Network in Real Time and Space Weather, Monograph NATO series, Effects of Space weather on Technology Infrastructure, 301-317, 2004.

Ruffolo, D., Bieber, J., Evenson, P., and Pyle, R.: Precursors to Forbush decreases and space weather predictions. Proc. 26 ICRC, 6, 440-443, 1999.

Tylka, A. J.: Solar energetic particles and space weather, Space Technology and Applications International Forum, Proc. AIP Conference Albuquerque, 552, 1185-1190, 2001.

Villoresi, G., Dorman, L. I., Iucci, N., and Ptitsyna, N. G: Cosmic ray survey to Antarctica and coupling functions for neutron component near solar minimum (1996-1997), 1. Methodology and data quality assurance, J. Geophys. Res., 105(A9), 21025 $21034,2000$.

Villoresi, G., Dorman, L. I., Ptitsyna, N. G., Iucci, N., and Tyasto, M. I.: Forbush-decreases as indicators of health-hazardous geomagnetic storms, Proc. 24th Intern. Cosmic Ray Conf. Rome, 4, 1106-1109, 1995. 